\title{
Probiotics for pediatric antibiotic-associated diarrhea: a meta-analysis of randomized placebo-controlled trials
}

\author{
Bradley C. Johnston, Alison L. Supina, Sunita Vohra
}

\section{ABSTRACT}

Background: Antibiotic treatment is known to disturb gastrointestinal microflora, which results in a range of clinical symptoms - most notably, diarrhea. This is especially important in children, for whom antibiotics are prescribed frequently. Although meta-analyses have been conducted to evaluate the ability of probiotics to prevent antibioticinduced diarrhea in the general population, little is known about which probiotic strains and doses might be of most benefit to children. Our objective in this study was to assess the efficacy of probiotics (of any specified strain or dose) for the prevention of antibiotic-associated diarrhea in children and to assess adverse events associated with the use of probiotics when coadministered with antibiotics to children.

Methods: A comprehensive search was performed of the major electronic databases (e.g., CENTRAL, MEDLINE, EMBASE, CINAHL, AMED) from their inception to January 2005. We also contacted experts and searched registries and meeting abstracts for additional relevant articles. Randomized controlled trials that compared probiotic treatment with placebo or no treatment, involving pediatric subjects less than 19 years of age were included. Two reviewers independently applied eligibility criteria and assessed the studies for methodological quality. Data were independently extracted by 2 reviewers and analyzed via the standard Cochrane methodology.

Results: Six studies were included (total $n=707$ patients). The combined results, analyzed with a per-protocol method that reported on the incidence of diarrhea during antibiotic treatment, showed significant benefit for the use of probiotics over placebo (relative risk [RR] $0.43,95 \%$ confidence interval $\left.[\mathrm{CI}] 0.25-0.75, \mathrm{I}^{2}=70.1 \%\right)$. In contrast, results from intention-to-treat analysis were nonsignificant overall (RR $1.01,95 \% \mathrm{Cl} 0.64-1.61$ ). Subgroup analysis on 4 studies that provided at least 5 billion single-strain colony-forming units (CFUs) daily (range 5.5-40 $\times 10^{9}$ Lactobacillus GG, L. sporogens or Saccharomyces boulardii) showed strong evidence with narrow $\mathrm{Cls}$ for the preventative effects of probiotics for antibiotic-associated diarrhea (RR $0.36,95 \% \mathrm{Cl} 0.25-0.53$, $\mathrm{I}^{2}=3 \cdot 5 \%$ ). No serious adverse events were reported.

Interpretation: The potential protective effects of probiotics to prevent antibiotic-associated diarrhea in children do not withstand intention-to-treat analysis. Before routine use is recommended, further studies (with limited losses of sub- jects to follow-up) are merited. Trials should involve those probiotic strains and doses with the most promising evidence (i.e., Lactobacillus GG, L. sporogens or S. boulardii at $5^{-40} \times 10^{9}$ CFUs daily).

CMAJ 2006;175(4):377-83

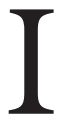
n 2003, the Canadian Paediatric Society released prescription information from administrative databases for over I 000 ooo pediatric claimants. Among the 20 most frequently prescribed drugs, I4 were antibiotics; $76 \%$ of children in the study population had been given a least 1 prescription for antibiotics in I999-2000. ${ }^{1}$

Almost all antibiotic treatments may disturb the colonization resistance of gastrointestinal flora and cause a range of clinical symptoms, most notably diarrhea. Clostridium difficile diarrhea, however, which is among the most serious of the adverse events related to antibiotic-associated diarrhea (AAD), occurs most often in older, immunocompromised adults who have been admitted to hospital. ${ }^{2}$ In the general population, $\mathrm{AAD}$ varies in incidence from $5 \%$ to $62 \%$, and in timing, from at the initiation of therapy to as long as 2 months after the end of treatment. ${ }^{2-4}$ Among children who receive broad-spectrum antibiotics, reported incidences of diarrhea vary from $\mathrm{II} \%$ to $62 \% .^{4-6}$ Although the World Health Organization (WHO) defines AAD as 3 or more abnormally loose bowel movements in a 24-hour period, definitions used in pediatric and adult trials have varied from $\mathrm{I}$ to 3 abnormally loose stools per $24-48$ hours. ${ }^{7,8}$

The term probiotic refers to a product or preparation containing viable, defined microorganisms in numbers thought to be sufficient to alter the host's microflora (by implantation or colonization) and thereby exert beneficial effects. ${ }^{9}$ The rationale behind probiotic administration is based on reinoculation with specific probiotic strains to normalize unbalanced indigenous microflora. Two meta-analyses of trials of probiotics and prevention of $\mathrm{AAD}$ in the general population have been completed, with results in favour of probiotic coadministration with antibiotics (odds ratio ${ }^{8} 0.37,95 \%$ confidence interval [CI] 0.26-0.53; risk ratio ${ }^{10}[\mathrm{RR}] 0.40$, 95\% CI 0.280.57); a third review, however, found insufficient evidence for the routine clinical use of probiotics to prevent or treat $C$. difficile-associated diarrhea in adults. ${ }^{11}$ Safety does not appear to be a concern in healthy individuals, although serious infections (e.g., bacteremia, meningitis, endocarditis, pneumonia 
and deep abdominal abscesses) have been reported in neonates and in severely debilitated and immunocompromised patients. ${ }^{12-20}$

We conducted a systematic review of randomized controlled trials of probiotics (of any specified strain or dose) coadministered with antibiotics (any agent) to assess their safety and efficacy in the prevention of pediatric AAD.

\section{Methods}

A comprehensive search was conducted for studies of probiotics, irrespective of publication status or language. The databases searched included ISI's Web of Science (I9452005), MEDLINE (I966-2005), EMBASE (I980-2005), CINAHL (I982-2005), AMED (I985-2005), the Cochrane Central Register of Controlled Trials (CENTRAL, 2005, issue 1) and the Cochrane Complementary Medicine Field's Register of Controlled Trials. Conference proceedings and dissertation abstracts were searched through Ovid's Dissertation Abstracts (I980-2005), the Conference Papers Index (I9822005) and the OCLC Online Computer Library Center (1992-2005). We also searched the "grey" literature (i.e., studies not published or with limited distribution), including, among other venues, databases housed by the Chalmers Research Group (PedCAM), CISCOM (Centralised In-

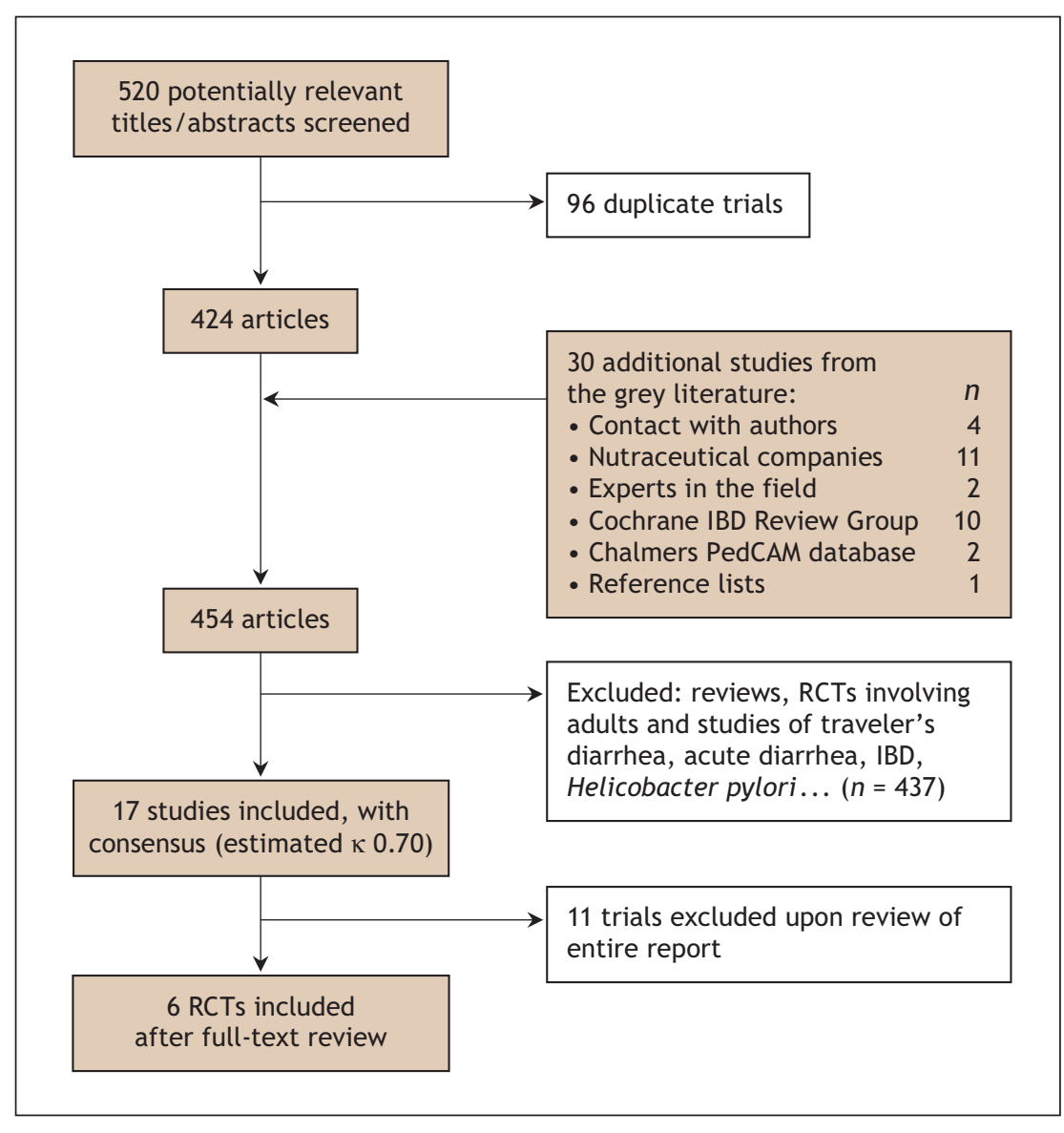

Fig 1: Flow diagram of search results. IBD = inflammatory bowel disease, RCTs = randomized controlled trials. formation Service for Complementary Medicine) and the Cochrane Inflammatory Bowel Disease Review Group; bibliographies of randomized controlled trials and review articles; American Gastroenterological Association meeting abstracts; and ongoing trials, located through the Current Controlled Trial Register. Inclusion criteria for our review were that the study be a randomized controlled trial; involve pediatric subjects (0-I8 years of age); use any dose of a specified probiotic of any strain, administered in conjunction with any antibiotic and compared with placebo; and report incidence outcomes for diarrhea (as defined by the study's authors) or adverse events.

Searches were first screened by 2 independent reviewers (B.C.J., A.L.S.) who looked at titles of papers and, when available, abstracts. The full text of each article selected was then retrieved and independently assessed by each of the 2 reviewers for inclusion according to prespecified selection criteria and for methodological quality. Each included study was evaluated with the (previously validated) 5 -point Jadad scale ${ }^{21}$ to assess its methods of randomization and double-blinding and the numbers of withdrawals and dropouts. Concealment of allocation was assessed as adequate, inadequate or unclear according to trial-design methodology described by Schulz and coauthors. ${ }^{22}$ Interrater reliability was assessed for both quality scales by using $\kappa$ statistics; disagreements were resolved by consensus.

Using a standardized data extraction form, the same 2 reviewers independently extracted data items, including methodological design, definition and diagnostic criteria for diarrhea, and inclusion and exclusion criteria for participants.

\section{Results}

From the primary electronic databases, we identified a total of 520 studies (EMBASE 277, MEDLINE I56, CENTRAL 38, Web of Science 32, CINAHL 17, AMED o). Thirty additional citations were identified from the grey literature (Fig. I). Using prespecified inclusion criteria, the independent reviewers identified 17 trials for full review. Upon full text review, 6 relevant studies $(5$ in English, ${ }^{7,23-26} 1$ in Italian $^{4}$ ) were included. Appendix I presents details on the II studies excluded. ${ }^{27-38}$ Interrater agreement for inclusion and exclusion was very good (estimated $\kappa 0.70) .{ }^{27}$ The 6 included studies enrolled a total of 836 participants (408 for treatment and 428 as control subjects, which totalled 707 after losses to followup). In each trial, participants were administered probiotics at the onset of antibiotic treatment; daily probiotic treatment was continued until the completion of the antibiotic course (7-I4 days). All 6 studies provided data on the incidence of pediatric di- 
arrhea. A detailed summary of the studies included can be found in Table I.

The overall pooled results with random-effects per-protocol analysis showed that the use of probiotics produced a significant reduction in the incidence of AAD (Fig. 2). The number needed to treat (NNT) in order to prevent I case of diarrhea was found to be 6 patients (NNT 5.74, 95\% CI 4.36-I3.08). Statistical heterogeneity, however, was moderate $\left(\mathrm{I}^{2}=70 \%\right.$; $\mathrm{I}^{2}$ represents the percentage of variability due to between-study variability). ${ }^{39}$ A sensitivity analysis that incorporated extremecase intention to treat (ITT) ${ }^{40}$ showed nonsignificant results overall (Fig. 3).

None of the 6 studies reported a definition of what constituted an adverse event. Three $e^{23,24,26}$ of the 4 studies that did monitor for adverse events reported that none occured. The fourth ${ }^{7}$ reported 14 minor adverse events (including rash, gas, vomiting, increased phlegm and chest pain) but not the group (treatment or control) in which they occurred.

Jadad quality scores for the 6 studies are shown in Table I. The mean score of 3.3 indicated good quality overall, ${ }^{21}$ whereas results for concealment of treatment allocation were mixed. ${ }^{22}$ Since there was full agreement between the 2 reviewers for both the Jadad and Shulz quality scales, $\kappa$ statistics were not calculated.

Two a priori subgroups were analyzed with respect to the primary outcome, incidence of diarrhea: probiotic strain and probiotic dose. Two of the 6 trials administered identical probiotic strains - Lactobacillus GG (L. casei spp rhamnosus). ${ }^{25,26}$ The summary statistics from these 2 studies $(n=307)$ provided statistical significance for a protective effect $(\mathrm{RR}=$ 0.29) of the Lactobacillus $G G$ strain (Fig. 4).

The dose-based subgroup analyses compared primary outcomes for daily doses of $5 \times 10^{9} \mathrm{CFUs}$ and above with those for lesser doses. The 4 studies that provided children with $5 \cdot 5-40$ $\times \mathrm{IO}^{9}$ bacteria or yeast cells per day showed evidence for the preventative effects of probiotics ( $R R$ o.36, 95\% CI 0.25$0.53),{ }^{4,24-26}$ whereas those for the single study that provided a substantially lower daily dose, $2 \times 10^{9}$ probiotic bacteria, ${ }^{7}$ were nonsignificant (Fig. 5). A $\chi^{2}$ test for potential dose-related heterogeneity ( 4 studies providing $\geq 5 \times \mathrm{IO}^{9} \mathrm{CFUs} / \mathrm{d}$ v. 1 study providing $\left.<5 \times 10^{9} \mathrm{CFUs} / \mathrm{d}\right)$ was significant $(p=0.002)$ and may explain the moderate statistical heterogeneity $\left(\mathrm{I}^{2}=70 \%\right)$ observed for the the incidence of diarrhea ${ }^{41}$ (Fig. 2, Fig. 5).

\section{Interpretation}

This systematic review of probiotic trials, which was focused on pediatric patients with $\mathrm{AAD}$, incorporated 3 trials ${ }^{4,23,24}$ not included in previous meta-analyses of probiotics for AAD in the general population. ${ }^{8,10}$ Yet another review evaluated probiotics for the prevention and treatment of $C$. difficile-associated diarrhea in adults. ${ }^{11}$ Of the 6 pediatric trials we included, 2 assayed stool samples for $C$. difficile. ${ }^{24,25}$ Since $26 \%-50 \%$ of AAD may be attributed to $C$. difficile, ${ }^{2}$ future trials should better address the efficacy of probiotics in preventing pediatric $\mathrm{AAD}$ caused by enteropathogens, particularly $C$. difficile.

Per-protocol pooled results of the 6 studies we reviewed yielded favourable results for the efficacy of probiotics (RR 0.43 , 95\% CI 0.25-0.75); treatment of 6 patients (NNT 5.7, $95 \%$ CI 4.4-I3.I) should prevent I case of diarrhea. Our findings are consistent with those of 2 earlier meta-analyses involving the general population: one by D'Souza and colleagues, ${ }^{8}$ who used a per-protocol analysis (odds ratio 0.37 , 95\% CI 0.26-0.53), and another by Cremonini and coauthors $^{10}$ (RR 0.40, 95\% CI 0.27-0.57), who excluded trials with losses to follow-up of $15 \%$ or more.

Table 1: Characteristics of studies included in the meta-analysis

\begin{tabular}{|c|c|c|c|c|}
\hline Study & $\begin{array}{l}\text { No of } \\
\text { participants } \\
\text { (loss to FU); } \\
\text { age range* }^{*}\end{array}$ & $\begin{array}{l}\text { Probiotic (antibiotic) interventions } \\
\text { with dosages, if reported }\end{array}$ & $\begin{array}{l}\text { Definition of diarrhea } \\
\text { for purposes of incidence }\end{array}$ & $\begin{array}{l}\text { Study quality: } \\
\text { Jadad score } † \\
\text { (allocation } \\
\text { concealment) }\end{array}$ \\
\hline $\begin{array}{l}\text { Arvola } \\
1999^{25}\end{array}$ & $\begin{array}{c}167(48) \\
2 \mathrm{wk}-12 \mathrm{yr}\end{array}$ & $\begin{array}{l}\text { Lactobacillus GG, } 20-40 \text { billion CFU/d for } \\
7-14 \text { d (antibiotics not specified) }\end{array}$ & $\begin{array}{l}\geq 3 \text { watery or loose stools per day } \\
\text { for } \geq 2 \text { consecutive days }\end{array}$ & 2 (unclear) \\
\hline $\begin{array}{l}\text { Jirapinyo } \\
2002^{23}\end{array}$ & $\begin{array}{cc}18 \quad(0) \\
1-36 \mathrm{mo}\end{array}$ & $\begin{array}{l}\text { L. acidophilus, Bifidobacterium infantis } \\
7 \mathrm{~d} \text { of probiotic treatment, dose not stated } \\
\text { (cefprozil, ampicillin, gentamycin, cloxacillin) }\end{array}$ & Not reported & 2 (unclear) \\
\hline $\begin{array}{l}\text { Kotowski } \\
2005^{24}\end{array}$ & $\begin{array}{c}269(23) \\
5 \mathrm{mo}-15 \mathrm{yr}\end{array}$ & $\begin{array}{l}\text { Saccharomyces boulardii } 10 \text { billion CFU/d } \\
\text { for 7-9 days (a mixture of antibiotics) }\end{array}$ & $\begin{array}{l}\geq 3 \text { loose or watery stools daily for } \\
\geq 48 \mathrm{~h} \text {, during or } \leq 2 \mathrm{wk} \text { after therapy }\end{array}$ & 5 (adequate) \\
\hline $\begin{array}{l}\text { LaRosa } \\
2003^{4}\end{array}$ & $\begin{array}{c}120(10) \\
\text { Mean age } 6.6 \mathrm{yr}\end{array}$ & $\begin{array}{l}\text { L. sporogens } 5.5 \text { billion CFU/d for } 10 \mathrm{~d} \\
\text { (amoxicillin, amoxicillin + clavulanate, } \\
\text { cephalosporin, erythromycin) }\end{array}$ & $\begin{array}{l}\geq 2 \text { liquid stools over } 24 \mathrm{~h} \text { during } \\
\text { study period }\end{array}$ & 4 (unclear) \\
\hline
\end{tabular}

Note: $F U$ = follow-up, CFU = colony-forming units.

*Unless otherwise specified.

†Score $<3$ indicates a study of poor quality; 5 indicates a study of maximal quality. 
We also analyzed incidence results for AAD with a more conservative extreme-case ITT analysis, the results of which were nonsignificant. A sensitivity analysis suggested that 2 studies with extreme losses to follow-up (37\% and $29 \%{ }^{25}$ ) accounted for why results did not withstand ITT analysis. In 1 study, ${ }^{25}$ these losses may have been related to feasibility issues; a 3-month follow-up with families, for example, was difficult to accomplish (Dr. Taina Arvola, personal communication, 2005). By acceptance that noncompliance and protocol deviations are likely to occur in real clinical situations, ITT analysis preserves the integrity of randomization, enhances the external validity of the results and (by controlling for the removal of noncompliers from the analysis) dissuades overoptimistic statements of the efficacy of an intervention. ${ }^{42}$ For this reason, it is the preferred analytic approach for pragmatic trials of effectiveness. ${ }^{43}$ However, ITT analysis is not robust if rates of loss to follow-up are high. Since our review involved the meta-analysis of explanatory rather than pragmatic trials, and since 3 trials ${ }^{4,7,25}$ of the 6 examined had high losses to fol- low-up (18\%-37\%), the validity of ITT analysis in this review can be questioned. We chose instead to focus on the per-protocol analysis, as other authors ${ }^{8,11}$ in this field have done.

The statistical heterogeneity we found was notable: moderate (70\%) in the per-protocol analysis (Fig. 2) and large $(78 \%)$ in the ITT analysis (Fig. 3 ). Nonetheless, the quantification of heterogeneity is one component of a wider investigation of variability across studies, perhaps the most important being diversity in clinical trials, which we explored via subgroup analysis. ${ }^{39}$ Our subgroup analyses (based on perprotocol analysis) explored the potential reasons for statistical heterogeneity, including dose and strain.

No dose-ranging studies have been reported to determine the minimal effective dose of a probiotic in preventing AAD. A priori, because we wanted to separate trials of subtherapeutic doses from the others, we chose for subgroup analysis daily doses of 5 billion CFUs and above versus lesser doses. Although dosage recommendations on the labels of probiotic products available in Canadian health food stores varies from I billion to 40 billion CFUs per day, doses at the lower end of this range might not colonize the intestine. ${ }^{44}$ Since clinical effectiveness has been demonstrated at 5.5 billion CFUs daily, we chose 5 billion CFUs per day as our cut-off. ${ }^{4}$

Our subgroup analyses provided preliminary evidence that probiotic dose may be responsible for the clinical and thus statistical heterogeneity observed $\left(\mathrm{I}^{2}=76 \%\right)$. A perprotocol subgroup analysis of 4 studies that provided $\geq 5$ billion CFUs daily to study participants (5.5-40 $\times$ IO $^{9}$ single-strain probiotic CFUs per day of Lactobacillus $G G$, L. sporogens or Saccharomyces boulardii) showed strong evidence for the preventative effects of probiotics ( $\mathrm{RR}=0.36,95 \% \mathrm{CI} 0.25-$ $\left.0.53, \mathrm{I}^{2}=3.5 \%\right)$. Our $\chi^{2}$ test for potential dose-related heterogeneity (Fig. 5) showed significance and may explain the moderate statistical heterogeneity observed (Fig. 2). When included trials that used the same probiotic intervention underwent subgroup analysis for probiotic strain (Fig. 4), the 2 trials of Lactobacillus GG (10-40 × I0 $\left.^{9} \mathrm{CFUs} / \mathrm{d}\right)$ demonstrated almost no statistical heterogeneity with pooled results, which significantly favoured probiotics (RR 0.29, 95\% CI o.I5$0.57) .{ }^{25,26}$ Since heterogeneity dropped substantially when data on identical strains (Lactobacillus $G G$, Fig. 4) and on similar doses were
Fig 3: Incidence of antibiotic-associated diarrhea - intention-to-treat analysis. The analysis showed a nonsignificant difference between probiotics and placebo (z score) and statistically significant heterogeneity. 
combined (Fig. 5), we speculated that the differences in probiotic strain and dose were plausible reasons for the observed heterogeneity of the pooled results.

With respect to safety, no trials reported a serious adverse event. Our meta-analysis found no significant differences in the incidence of any adverse events between treatment and control groups, although no trial defined a priori what was considered to be an adverse event/reaction.

Two additional issues are worthy of further consideration during review of pediatric trials of AAD: the effect of age and the definition of $\mathrm{AAD}$. Whereas the 2 meta-analyses done previously included both children and adults, with the majority of included trials involving adults, our review was restricted to the pediatric population; the included patients ranged in age from 2 weeks to 15 years. ${ }^{8,10}$ The difficulty in accurately measuring frequency or consistency of diarrhea in diapered infants versus in children and adolescents may make it difficult to detect differences. For example, infants not only have bowel movements more frequently, ${ }^{45}$ but their stools may also be looser in consistency than those of older children, which makes infant stools more difficult to distinguish from diarrhea. Furthermore, it can be problematic to detect the frequency and consistency of stools accurately in diapered infants. Although randomized trials may evenly distribute infants between treatment and control groups so as to eliminate this potential confounder, the considerable differences in bowel habits of children at various ages may obscure the effect of probiotics in this population, and should be taken into consideration when planning pediatric trials. ${ }^{46}$

Second, the definitions of AAD in included studies varied, including " 1 or more abnormally loose bowel movements per day throughout the study period,"7 as opposed to "at least 3 watery or loose stools per day for at least 2 consecutive days." 25 One trial ${ }^{23}$ did not provide a definition at all. A survey of clinicians and

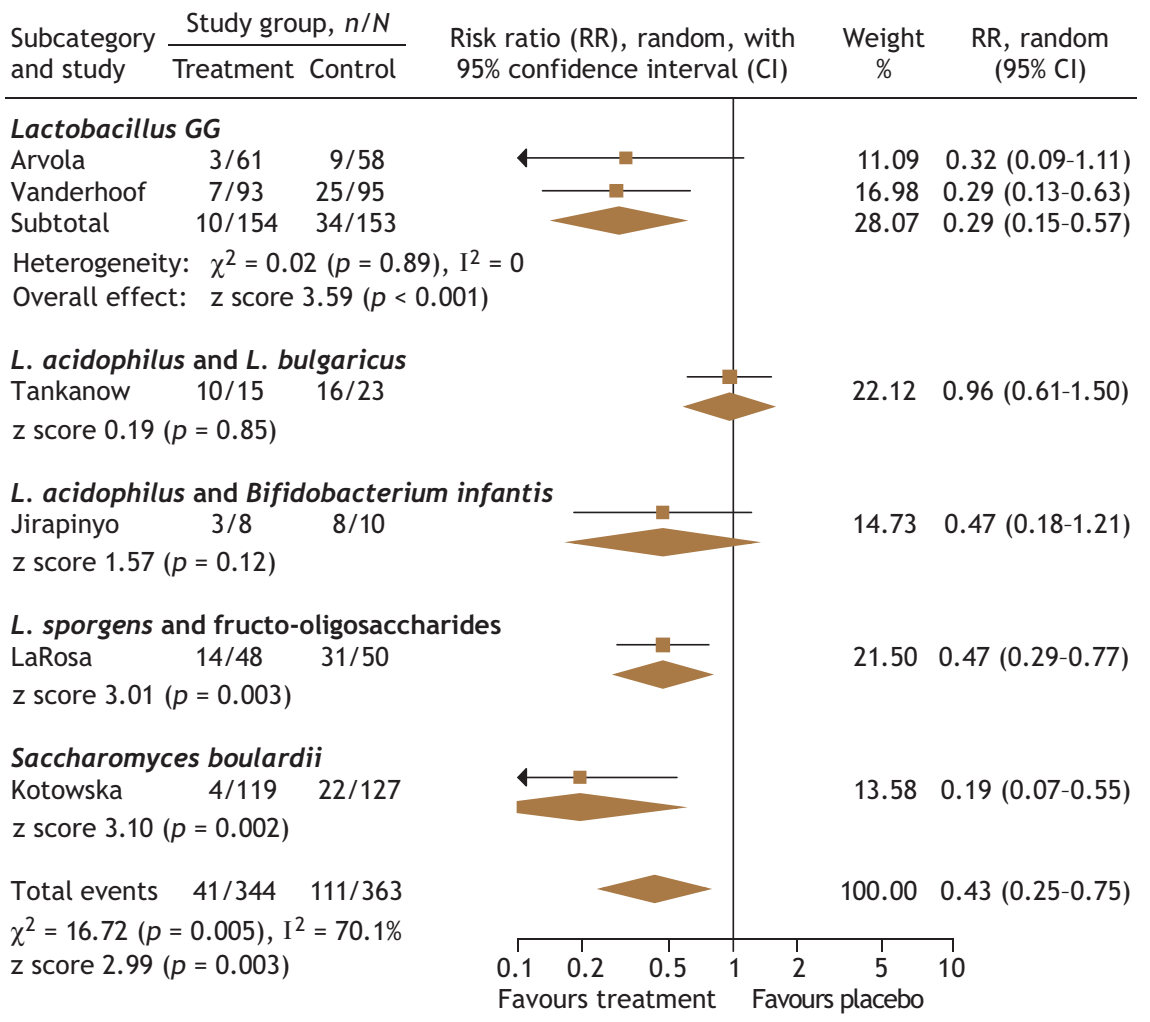

Fig 4: Incidence of antibiotic-associated diarrhea, by the probiotic strain administered. Both the overall heterogeneity $\left(\mathrm{I}^{2}\right)$ and the difference between probiotics and placebo (z score) were statistically significant. For the combined results of 2 trials of Lactobacillus $G G$, the heterogeneity was nonsignificant, with a significant difference in overall effect compared with placebo.

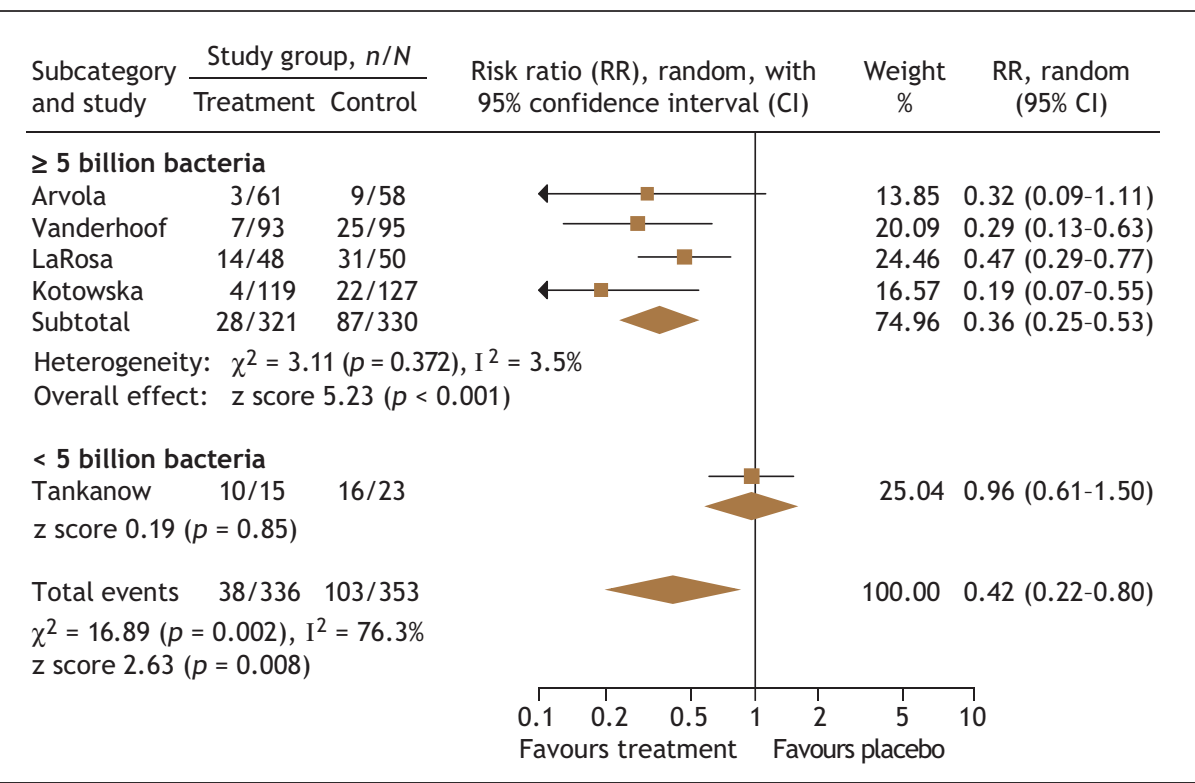

Fig 5: Incidence of antibiotic-associated diarrhea, by probiotic dose. For the 4 studies administering daily doses of at least $5 \times 10^{9}$ colony-forming units (CFUs), the overall effect differed significantly from that of placebo, with minimal heterogeneity. When all data were pooled, however, heterogeneity also became statistically significant. 
parents may be helpful to document what is considered a clinically meaningful reduction in AAD and the point at which parents and clinicians would be likely to consider the coadministration of a probiotic.

This systematic review has several strengths. Compared with previous reviews of probiotics for AAD in the general population, our search strategy was more comprehensive. Although we had too few trials reporting on the incidence of pediatric diarrhea $(n=6)$ to properly analyze for publication bias, ${ }^{47}$ several strategies were implemented to control this. We conducted explicit searches in multiple databases, and considered any trial irrespective of publication format (e.g., abstracts) or language. We also conducted a priori subgroup (probiotic strain, dose) and sensitivity analyses (per-protocol v. ITT) to further explore our hypotheses, and looked for heterogeneity as a result of pooling.

Our review also had a number of limitations. We did not search the European Gastroenterology Week conference proceedings, a potential home for relevant trials; nor did we include trials that assessed probiotics versus standard conventional care for AAD (Appendix I). ${ }^{31,32}$ Although these effectiveness trials may have provided additional relevant data, we were interested in comparing the efficacy of probiotics with placebo via explanatory trials. ${ }^{48}$ Finally, some readers may question the pooling of different probiotic strains. In keeping with our justification for including the combinations of probiotic strains that were used in 2 trials included in this review (Tankanow and associates ${ }^{7}$ administered both L. acidophilus and L. bulgaricus; Jirapinyo and coworkers ${ }^{23}$ administered L. acidophilus with Bifidobacterium infantis), we pooled our overall results because the probiotics used share the recommended characteristics of a viable probiotic agent: nonpathogenic properties, the ability to survive transit through the gastrointestinal tract, adherence to intestinal epithelium, colonization in the intestinal tract, production of antimicrobial substances, and a good shelf life in food or powdered form. ${ }^{49}$

In sum, the potential protective effects of probiotics to prevent pediatric AAD did not withstand ITT analysis. Future studies should engage probiotic strains and doses with the most promising evidence (e.g., Lactobacillus GG, L. sporogens or $S$. boulardii at $\left.5-40 \times 10^{9} \mathrm{CFUs} / \mathrm{d}\right)$; determine the effect of age (i.e., infants v. older children) and ensure that adverse events are reported and losses to follow-up are limited. Trials would also benefit from a validated primary outcome measure for $\mathrm{AAD}$ that is sensitive to change and reflects what stool frequencies and consistencies have importance in the eyes of clinicians, parents and children. The current data are promising, but inconclusive; it is premature to draw a conclusion about the efficacy and safety of probiotics for pediatric AAD until such trials are completed. For clinicians contemplating the use of probiotics in their pediatric practice, the use of Lactobacillus GG, L. sporogens or S. boulardii at a dose of 5-40 billion CFUs per day appears to hold promise as an option for coadministration with antibiotics, and no serious adverse events were reported in the included studies. Nevertheless, safety is better assessed in population-based samples than in the relatively small numbers exposed in randomized controlled trials.
This article has been peer reviewed.

From the Complementary and Alternative Research and Education (CARE) Program (Johnston, Vohra), Stollery Children's Hospital, Department of Pediatrics, University of Alberta, Edmonton; and the Department of Community Health Sciences, Health Sciences Centre, and the Centre for Health and Policy Studies (Supina), University of Calgary, Calgary, Alta.

Competing interests: Bradley Johnston has received study product from Culturelle Inc., the manufacturer of Lactobacillus $G G$, for evaluation in pediatric patients with irritable bowel syndrome.

Contributors: Bradley Johnston wrote the study protocol, managed and participated in screening and quality assessment of and data extraction from articles, completed the statistical analyses, interpretated results, and drafted the manuscript. Alison Supina assisted with screening, data extraction and quality assessment. Sunita Vohra assisted with design of the methodology, statistical analyses and interpretation of results. All coauthors critically revised the article and approved the version to be published.

Acknowledgements: We thank Ellen T. Crumley for assistance with the electronic search, and Natasha Wiebe and Ben Vandermeer for fruitful discussions on statistical methods and interpretations.

Bradley Johnston holds a Sick Kids Foundation Duncan L. Gordon Fellowship. Sunita Vohra is an Alberta Heritage Foundation for Medical Research Population Health Investigator and is the recipient of a Canadian Institute of Health Research New Investigator Award.

\section{REFERENCES}

I. Khaled A, Ahmad F, Brogan T, et al. Prescription medicine use by one million Canadian children. Paediatr Child Health 2003;8(Suppl A):43A-5A.

2. McFarland LV. Epidemiology, risk factors and treatments for antibiotic-associated diarrhea [review]. Dig Dis 1998;16:292-307.

3. Wistrom J, Norrby SR, Myhre EB, et al. Frequency of antibiotic-associated diarrhoea in 2462 antibiotic-treated hospitalized patients: a prospective study. J Antimicrob Chemother 200I;47:43-50.

4. LaRosa M, Bottaro G, Gulino N, et al. Prevention of antibiotic-associated diarrhea with Lactobacillus sporogens and fructo-oligosaccharides in children: a multicentric double-blind vs. placebo study. Minerva Pediatr 2003;55:447-52.

5. Turck D, Bernet JP, Marx J, et al. Incidence and risk factors for of oral antibioticassociated diarrhea in an outpatient pediatric population. $J$ Pediatr Gastroenterol Nutr 2003;37:22-6.

6. Elstner CL, Lindsay AN, Book LS, et al. Lack of relationship of Clostridium difficile to antibiotic-associated diarrhea in children. Pediatr Inf Dis 1983;2:364-6.

7. Tankanow RM, Ross MB, Ertel IJ, et al. A double-blind, placebo-controlled study of the efficacy of Lactinex in the prophylaxis of amoxicillin-induced diarrhea. DICP I990;24:382-4.

8. D'Souza AL, Rajkumar C, Cooke J, et al. Probiotics in the prevention of antibiotic associated diarrhea: meta-analysis. $B M J$ 2002;324:136I-6.

9. Havenaar R, Huis in 't Veld JHJ. Probiotics: a general view. In: Wood, BJB, editor. Lactic acid bacteria in health and disease. Vol I of the series, The lactic acid bacteria. Amsterdam: Elsevier Applied Science Publishers; 1992.

Io. Cremonini F, Di Caro S, Nista EC, et al. Meta-analysis: the effect of probiotic administration on antibiotic associated diarrhea. Aliment Pharmacol Ther 2002;16: I46I-7.

II. Dendukuri N, Costa V, McGregor M, et al. Probiotic therapy for the prevention and treatment of Clostridium difficile-associated diearrhea: a systematic review. CMA 2005;173(4):167-70.

I2. Land MH, Rouster-Stevens K, Woods CR, et al. Lactobacillus sepsis associated with probiotic therapy. Pediatrics 2005;II5:I78-8I.

I3. Salminen MK, Rautelin H, Tynkkynen S, et al. Lactobacillus bacteremia, clinical significance, and patient outcome, with special focus on probiotic L. rhamnosus GG. Clin Infect Dis 2004;38:62-9.

I4. Mackay AD, Taylor MB, Kibbler CC, et al. Lactobacillus endocarditis caused by a probiotic organism. Clin Microbiol Infect I999;5:290-2.

I5. Rautio M, Jousimies-Somer H, Kauma H, et al. Liver abscess due to a Lactobacillus rhamnosus strain indistinguishable from L. rhamnosus strain GG. Clin Infect Dis I999;28:1159-6o.

I6. Piarroux R, Millon L, Bardonnet K, et al. Are live Saccharomyces yeasts harmful to patients? Lancet 1999;353:185I-2.

I7. Salminen S, Wright AV, Morelli L, et al. Demonstration of safety of probiotics: a review. Int J Food Microbiol I998;44:93-106.

I8. Saxelin M, Chuang NH, Chassy B, et al. Lactobacilli and bacteria in southern Finland, I989-1992. Clin Infect Dis 1996;22:564-6.

I9. Hata D. Meningitis caused by bifidobacteria in an infant. Pediatr Infect Dis J I988; 7:669-7I.

20. Sussman J, Baron E, Goldberg S, et al. Clinical manifestations of Lactobacillus endocarditis: report of a case and review of the literature. Rev Infect Dis 1986;8:77I-6. 
2I. Jadad AR, Moore RA, Carrol D, et al. Assessing the quality of reports of randomized clinical trials: Is blinding necessary? Control Clin Trials 1996;17:I-I2.

22. Schulz KF, Chalmers I, Hayes RJ, et al. Empirical evidence of bias: dimensions of methodological quality associated with estimates of treatment effects in controlled trials. JAMA I995;273:408-I2.

23. Jirapinyo P, Densupsoontorn N, Thamonsiri N, et al. Prevention of antibiotic-associated diarrhea in infants by probiotics. JMed Assoc Thai 2002;85(Suppl 2):S739-42.

24. Kotowska M, Albrecht P, Szajewska H. Saccaromyces boulardii in the prevention of antibiotic-associated diarrhea in children: a randomized double-blind placebocontrolled trial. Aliment Pharmacol Ther 2005;21:583-90.

25. Arvola T, Laiho K, Torkkeli S, et al. Prophylactic Lactobacillus GG reduces antibiotic-associated diarrhea in children with respiratory infections: a randomized study. Pediatrics 1999;104:e64

26. Vanderhoof J, Whitney DB, Antonson DL, et al. Lactobacillus GG in the prevention of antibiotic-associated diarrhea in children. J Pediatr 1999;135:564-8.

27. Landis JR, Koch GG. An application of hierarchical kappa-type statistics in the assessment of majority agreement among multiple observers. Biometrics 1977;33: 363-74.

28. Chapoy P. Treatment of acute infantile diarrhea: controlled trial of Saccharomyces boulardii [in French]. Ann Pediatr (Paris) I985;32:56I-3.

29. Erdeve O, Tiras U, Dallar Y. The probiotic effect of Saccharomyces boulardii in a pediatric age group. J Trop Pediatr 2004;50:234-8.

3o. Seki H, Shiohara M, Matsumura T, et al. Prevention of antibiotic-associated diarrhea in children by Clostridium butyricum MIYAIRI. Pediatr Int 2003;45:86-90.

3I. Benhamou PH, Berlier P, Danjou G, et al. Antibiotic-associated diarrhea in children: a computer-monitored double-blind outpatient trial comparing a protective and a probiotic agent [in French]. Med Chir Dig 1999;28:163-8.

32. Schrezenmeir J, Heller K, McCue M, et al. Benefits of oral supplementation with and without synbiotics in young children with acute bacterial infections. Clin Pediatr (Phila.) 2004;43:239-49.

33. Michielutti F, Bertini B, Presciuttini B, et al. Clinical validation of a new oral bacteria therapy in pediatric patients with acute diarrhea [in Italian]. Minerva Med I996; 87:545-50.

34. Thomas MR, Litin SC, Osmon DR, et al. Lack of effect of Lactobacillus GG on antibiotic-associated diarrhea: a randomized, placebo-controlled trial. Mayo Clin Proc 2001;76:883-9.

35. Witsell DL, Garrettt CG, Yarbrough WG, et al. Effect of Lactobacillus acidophilus on antibiotic-associated gastrointestinal morbidity: a prospective randomized trial. JOtolaryngol $1995 ; 24: 230-3$

36. Siitonen S, Vapaatalo H, Salminen S, et al. Effect of Lactobacillus GG yoghurt in prevention of antibiotic associated diarrhoea. Ann Med I990;22:57-9.

37. Zoppi G, Cinquetti M, Benini A, et al. Modulation of the inestinal ecosystem by probiotics and Lactulose in children during treatment with ceftriaxone. Curr Ther Res Clin Exp 2001;62:418-35.

38. Contardi I. Oral bacterial therapy for the prevention of antibiotic-induced diarrhea in pediatrics [in Italian]. Clinica Terapeutica I991;136:409-13.

39. Higgins JPT, Thompson SG, Deeks JJ, et al. Measuring inconsistency in metaanalyses. BMJ 2003;327:557-60.

40. Hollis S, Campbell F. What is meant by intention to treat analysis? Survey of published randomized controlled trials. BMJ I999;319:670-4.

4I. Deeks JJ, Altman DG, Bradburn MI. Statistical methods for examining heterogeneity and combining results from several studies in meta-analysis. In: Egger M, Davey Smith G, Altman DG, editors. Systematic reviews in health care. London: BMJ Books; 200I.
42. Heritier SR, Gebski VJ, Keech AC. Inclusion of patients in clinical trial analysis: the intention-to-treat principle. Med J Aust 2003;179:438-40.

43. Roland M, Torgerson DJ. Understanding controlled trials. What are pragmatic trials? BMJ I998;316:285.

44. Raza S, Graham SM, Allen SJ, et al. Lactobacillus $G G$ promotes recovery from acute nonbloody diarrhea in Pakistan. Pediatr Infect Dis J I995;I4:I07-II.

45. Isolauri $\mathrm{E}$, Juntunen $\mathrm{M}$, Rautanen $\mathrm{T}$, et al. A human Lactobacillus strain (Lactobacillus casei sp strain $G G$ ) promotes recovery from acute diarrhea in children. Pediatrics I991;88:90-7.

46. Fontana M, Bianchi C, Cataldo F, et al. Bowel frequency in healthy children. Acta Paediatr Scand i989;78:682-4.

47. Cochrane Handbook for Systematic Reviews. Appendix 8a: Considerations and recommendations for figures in Cochrane reviews. Graphs of statistical data: funnel plots. 2003.

48. McMahon AD. Study control, violators, inclusion criteria and defining explanatory and pragmatic trials. Stat Med 2002;21:1365-76.

49. Goldin BR. Health benefits of probiotics. BrJ Nutr 1998;80:S203-7

Correspondence to: Dr. Sunita Vohra, Director, CARE Program, Stollery Children's Hospital, Associate Professor of Pediatrics, Department of Pediatrics, University of Alberta, Aberhart Centre \#I, Rm. 82I3, II402 University Ave. NW, Edmonton AB T6G 2J3; 780 407-3798; fax 780 407-2105; svohra@ualberta.ca

Appendix 1: Studies excluded after full-text review

Reason for exclusion Study

Nonpediatric study population

Thomas $2001^{34}$

Witsell $1995^{35}$

Siitonen $1990^{36}$

Study groups not randomly assigned

Seki $2003^{30}$

Chapoy $1985^{28}$

Probiotic compared to other than placebo:

- A nutritional drink

- Diosmectite, an antidiarrheal drug

Schrezenmeir $2004^{32}$ Benhamou $1999^{31}$

Acute diarrhea not associated with use of antibiotic therapy

Erdeve $2004^{29}$ Michielutti $1996^{33}$

Outcomes not particular to incidence of diarrhea or adverse events

Zoppi $2001^{37}$ Contardi $1991^{38}$

\section{LEADERSHIP}

$C M A J$ is a founding member of the International Committee of Medical Journal Editors, an organization that is devoted to ensuring the highest integrity in scientific publishing and is a driving force in the mandatory registration of clinical trials. 